\title{
Streptococcus pyogenes Sternoclavicular Septic Arthritis in a Healthy Adult
}

\author{
Radmila M. Savcic-Kos, MD; Padmavati Mali, MD; Ajit Abraham, MD; Meltiady Issa, MD; \\ Venu Rangu, MD; and Rana Nasser, MD
}

\begin{abstract}
Sternoclavicular septic arthritis is a rare infection, accounting for approximately I\% of septic arthritis in the general population. Staphylococcus aureus is the predominant etiologic agent, and it usually occurs in relatively young adults with some type of predisposition to infection. We report, to the best of our knowledge, the first case of group A streptococcal, sternoclavicular arthritis in a previously healthy 62-year-old male patient. We present a detailed history and physical examination, with laboratory findings, imaging studies, cultures, and therapy.
\end{abstract}

Keywords: Arthritis/infectious; Osteomyelitis; Sternoclavicular joint; Streptococcus pyogenes

$\mathrm{S}$ that accounts for approximately $1 \%$ of septic arthritis in the general population and $17 \%$ in intravenous drug users. ${ }^{1,2}$ It is most commonly caused by Staphylococcus aureus. ${ }^{1,3-5}$ This report illustrates the first case in which the pathogen is Group A streptococcus (GAS).

\section{Case Presentation}

A male patient, aged 62 years, with a remote past history of implanted pacemaker for carotid sinus hypersensitivity, was admitted to the hospital with flu-like symptoms. He complained of fatigue and a productive cough for 3 weeks without fever. He had been exposed to children with documented GAS pharyngitis but denied any sore throat. Oseltamivir and azithromycin had been given orally one day before admission at a local urgent care department. His condition did not improve, and he was hospitalized the next day. Other than pacemaker placement for carotid sinus hypersensitivity, his past medical history was unremarkable, and he denied intravenous drug use. Blood and throat cultures, monospot test, and Epstein-Barr Virus-IgM antibodies were negative. Azithromycin was continued

Corresponding Author: Radmila M. Savcic-Kos, MD; Medicine-Pediatrics; $1000 \mathrm{~N}$ Oak Avenue; Marshfield WI, 54449; Tel: (7I5) 387-5600; Fax: (7I5) 389-5757; Email: savcic-kos.radmila@marshfieldclinic.org intravenously. He was discharged after 2 days and asked to complete a 5-day course of this antibiotic.

Three days following discharge, his right shoulder became sore after lifting furniture, and he was treated in a chiropractor's office. He developed neck stiffness, worsening pain, and decreased range of motion of the right side of the neck. No other trauma or injury was reported. He was diagnosed with a rotator cuff tear and treated with cyclobenzaprine and acetaminophen with hydrocodone.

The patient was examined the following day by a sports medicine specialist who found redness and swelling of the right sternoclavicular joint (SCJ). Chest radiography did not reveal any pathologic findings in the right SCJ or clavicle. Computed tomographic (CT) scan of the chest demonstrated edema of the right sternocleidomastoid muscle, skin, subcutaneous tissues, fat of the supraclavicular fossa, the pectoralis major muscle, and the right part of the neck (figure 1). No bone destruction or muscle abscess was visualized. Ultrasound-guided SCJ aspiration was performed: a small amount of pinkish fluid was obtained, enough only for a

Received: July I, 2013

Revised: September 20, 2013

Accepted: October 8, 2013

doi: $|0.3| 2|/ \mathrm{cmr} .20| 3.1 \mid 85$ 


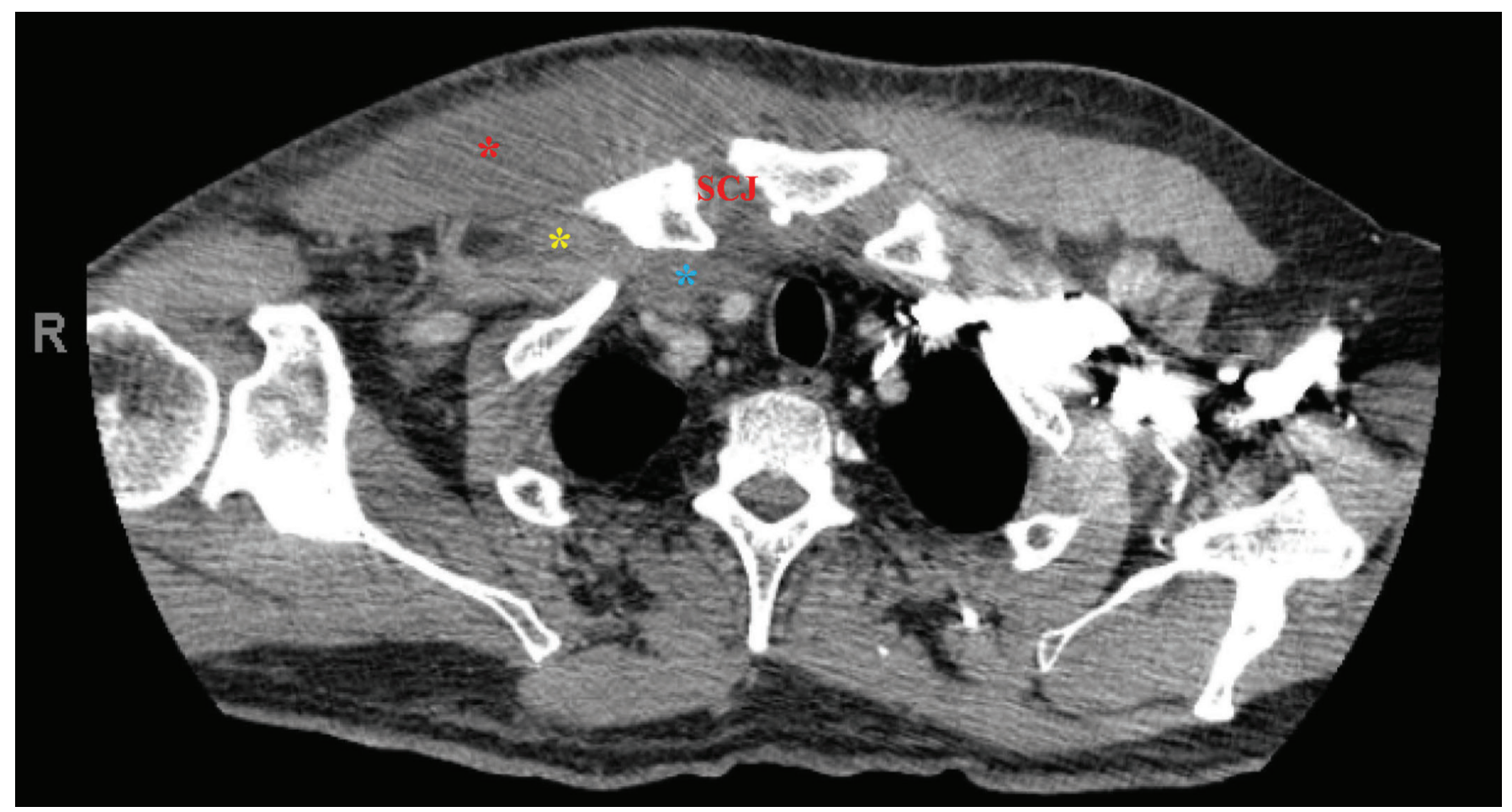

Figure 1. Computed tomography of the chest demonstrating swelling of the right pectoralis muscle (red star), soft (yellow star) and fat tissue (blue star).

culture. He was then hospitalized for management of right sternoclavicular infection.

Upon admission, he had right shoulder pain, weakness on external rotation, and decreased abduction to 20 degrees on active and 80 degrees on passive range of motion. Physical examination revealed temperature of $98.5^{\circ} \mathrm{F}$, heart rate of 77 beats $/ \mathrm{min}$, respiratory rate of 20 breaths $/ \mathrm{min}$, and blood pressure of 143/78 mmHg. The patient was alert and oriented. No axillary or cervical lymphadenopathy was palpated. The oropharynx was not erythematous, and his lungs were clear to auscultation. Heart sounds were normal without murmurs, rubs, or gallops. Chest wall examination revealed extensive swelling, warmth, tenderness, and erythema of the right sternoclavicular area (figure 2). Left-sided pacemaker site was intact, and Impingement and Hawkins tests were positive.

Laboratory tests revealed the following: white blood cell count $15.6 \times 10^{3} / \mathrm{uL}$ (reference range $4.5-13.5 \times 10^{3} / \mu \mathrm{L}$ ) with $2 \%$ bands, $77 \%$ segmented; hemoglobin $12.7 \mathrm{~g} / \mathrm{dL}$ (reference range 12.9-17.3 g/dL); platelets 620,000 (reference range $150,000-450,000)$. Electrolytes, blood urea nitrogen, venous lactate, creatinine, and liver function tests were within normal limits.

Incision and drainage of the SCJ and the head of the clavicle were performed shortly after admission, and a purulent drainage was evacuated. Pulse lavage irrigation was done, and vacuum-assisted closure (VAC) dressing of the wound was applied.
Group A streptococcus grew in the joint fluid and clavicle specimen, but blood cultures were negative. The patient was started on intravenous cefazolin $2 \mathrm{~g}$ every 8 hours. Because of his pacemaker, transthoracic echocardiography was obtained, and no vegetations were found. His local findings improved, and white blood cell count with differential count normalized, as did the C-reactive protein. Drainage from the wound significantly decreased, and he was discharged on day 7 with a wound VAC. He was to continue penicillin G, 18 million units by continuous 24 -hour infusion, for 4 weeks. Follow-up examination at the end of antibiotic therapy showed healing of the right SCJ incision site.

Four days after stopping the penicillin, the patient developed pain, redness, and swelling over the site of the pacemaker pocket in left upper chest. Ultrasound revealed cellulitis of the subcutaneous tissue of the chest wall, extending over the pacemaker. The pacemaker, along with wires, was removed. The wires were not cultured, but GAS grew from the drainage culture. There were no signs of endocarditis on echocardiography. The patient completed an additional 6 weeks of penicillin therapy (continuous infusion of intravenous penicillin $\mathrm{G}$ for 4 weeks and oral penicillin for 2 weeks), and the infection was eradicated.

\section{Discussion}

Sternoclavicular osteomyelitis is a relatively unusual infection among the general population. From analysis of six large series of septic arthritis, the SCJ was involved in only about $1 \%$ (8 out of 738 cases), ${ }^{6-9}$ but incidence is $17 \%$ among intravenous drug users. ${ }^{10,11}$ The SCJ can be infected by 

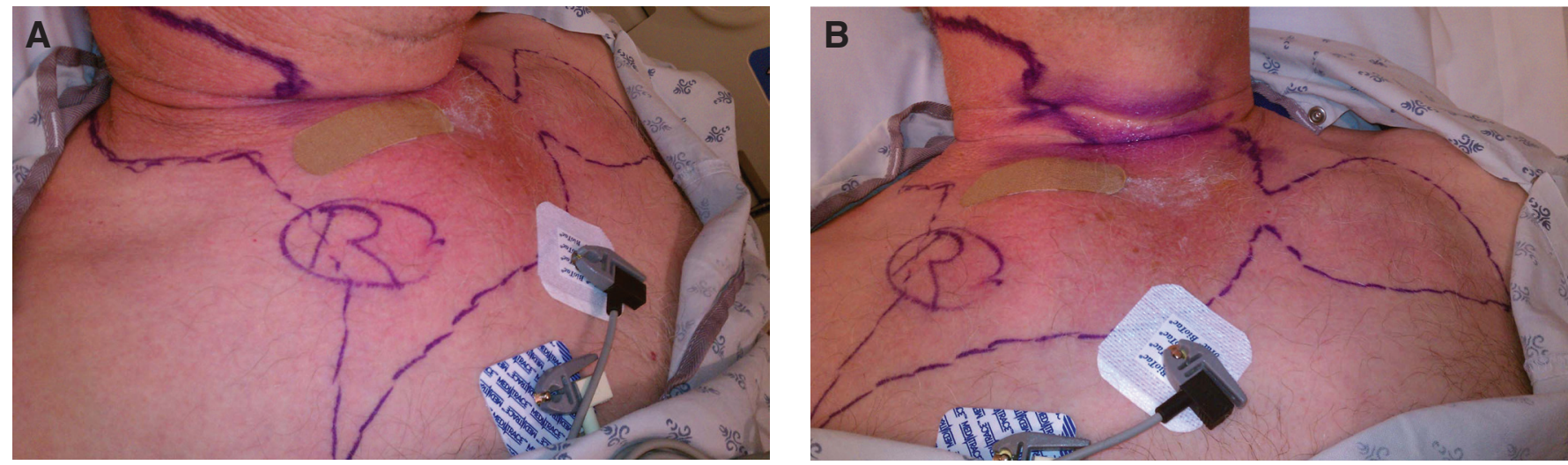

Figure 2. Chest wall examination revealed extensive swelling, warmth, tenderness, and erythema of the right sternoclavicular area. (A) side view; (B) front view.

bacterial seeding from a bloodstream infection with an average age of occurrence at 45 -years and more frequently in intravenous drug users. ${ }^{1}$ In the rest of the affected individuals, it is possible that they are more prone to minor trauma that facilitates bacteremic seeding. SCJ is a diarthrodial synovial joint (saddle-like) that allows rotation. ${ }^{12,13}$ Unlike the case of synovial joints, bacteria may not be efficiently cleared from SCJ. Ligamentous and capsular laxity changes with age, exposing both joints to greater strain, which may explain the rising incidence of arthritis with age. ${ }^{14}$ Sternoclavicular septic arthritis (SCSA) is less common in the elderly due to joint sclerosis..$^{15}$ Our patient was 62-years-old, with no history of drug abuse, and we thought the seeding was likely from his throat infection.

Sternoclavicular arthritis usually presents with an insidious onset of chest pain in the SCJ area (78\%) or referred pain in the ipsilateral neck and/or shoulder, accompanied by swelling and redness. It may also present with symptoms affecting the shoulder (24\%), and occasionally the neck (2\%). The median duration of symptoms at diagnosis is 14 days (range 2-6 weeks), which is longer than septic arthritis involving other joints. ${ }^{6}$ Fever and leukocytosis may not be present. Predisposing factors include diabetes mellitus, intravenous drug use, endocarditis, radiation therapy, immunosuppression, trauma, central venous catheter placement, surgery, renal failure, cirrhosis, portal hypertension, and cancer. ${ }^{1,3,7}$ Our patient presented with shoulder pain that was misdiagnosed as a rotator cuff tear. He also complained of neck pain, and the infection progressed to redness, swelling, and pain in the right SCJ.

A review of 180 cases of SCSA showed a predominance of right-sided SCJ involvement in both intravenous drug users $(54 \%)$ and non-drug users $(60 \%) .{ }^{1}$ In the same series, polyarticular arthritis was reported in $21 \%$ of cases and bilateral SCSA in 5\%. Because of the movement that occurs with shoulder motion, and because of the nerve supply for the SCJ, SCSA may cause referred pain to the shoulder. ${ }^{4}$ The SCSA innervation is by the medial supraclavicular nerve that comes from the superficial cervical plexus (C3 and $\mathrm{C} 4)$ and the subclavius nerve ( $\mathrm{C} 5$ and $\mathrm{C} 6$ ). These nerves provide innervation to the anteromedial shoulder and the anterior/ superior chest, which explains the radiation of pain to the shoulder. ${ }^{16}$ Furthermore, Hasset and Barnsley ${ }^{17}$ found that radiation of the pain from the SCJ to the jaw and elbow can be seen, which can mimic the presentation of a myocardial infarction. The presentation is usually subacute, as it was in our patient, and median duration of symptoms varies from 2 to 6 weeks. ${ }^{1,4}$ Fever was found in $52 \%$ to $65 \%$ of cases. ${ }^{1,18}$ Our patient had a low grade fever during his previous hospitalization.

Infections that may be implicated as a source of SCSA are pneumonia, cellulitis, endocarditis, urosepsis, spontaneous bacterial endocarditis, epidural or intra-abdominal abscess, gingivitis, and tuberculosis; although no risk factor was found in $23 \%$ of patients. ${ }^{1,18}$ In our case, the patient was healthy, without trauma, injury, immunosuppression, or intravenous drug use. The only preceding symptoms were from upper respiratory infection, and initial laboratory findings in our patient showed leukocytosis, neutrophilia, and elevated C-reactive protein. Leukocytosis is reported in $26 \%$ to $56 \%$ of such patients. ${ }^{1,18}$

The diagnosis of SCSA is established by joint fluid culture or a surgical specimen from joint/clavicle debridement, which is positive in most patients (77\%). ${ }^{1,19}$ S. aureus is responsible for approximately half of the cases (49\%), with other common pathogens reported as Pseudomonas aeruginosa (10\%), Brucella (7\%), and Escherichia Coli (5\%). Rare causes are caused by Bacteroides, ${ }^{4,7,20}$ Haemophilus influenzae, mycobacterium tuberculosis, and Mycobacterium aviumintracellulare. ${ }^{8,21}$ Our review of the literature using PUBMED and MEDLINE yielded no other references citing GASSCSA in immunocompetent, non-intravenous drug user, adults. One report documented polymicrobial polyarticular infection of the knee, elbow and shoulder with GAS and other pathogens. ${ }^{22}$ There was a French report of GAS sternoclavicular arthritis in an HIV (+) patient published in 1990..$^{23}$ Joint fluid and clavicle specimens from our patient grew $\beta$-hemolytic GAS. The negative blood culture may be explained by the fact that he was already on antibiotics. 
Bacteremia is more prevalent in patients with SCSA (62\%) than in other types of septic arthritis $(27 \%),{ }^{6}$ which supports the endovascular source of bacteremia, such as phlebitis of the subclavian vein in intravenous drug users. He had a history of febrile illness with associated symptoms of upper respiratory infection 2 weeks before presentation with the joint infection. Blood and throat cultures were negative, but the patient had already been started on antibiotics. We speculate that the infection originated as pharyngitis with resultant localized abscess formation and contiguous spread to the SCJ. Alternatively, spread of the infection may have been hematogenous, originating as a pharyngitis.

Imaging is also important for diagnosis of SCSA. Conventional radiography is not very helpful for evaluation of bone changes. ${ }^{419}$ Early plain radiology findings may demonstrate soft tissue swelling and cortical destruction of a bone. In later stages, periosteal reaction and sequestrum formation may be seen. CT is more sensitive in identifying early changes such as widened joint, irregular bony margins, and bony destruction. ${ }^{4}$ In later stages of infection, periosteal reaction, sequestra formation, and reactive sclerosis may be seen. No abnormal radiographic plain film findings were seen in the $\mathrm{SCJ}$ or clavicle in our patient. Chest $\mathrm{CT}$ demonstrated edema of the right sternocleidomastoid and pectoralis major muscles, as well as in the surrounding skin and soft tissue. Another imaging modality is technetium- $99 \mathrm{~m}$ that may show changes only hours after the onset of infection. ${ }^{4}$ Lastly, magnetic resonance imaging (MRI) is very useful, with reported sensitivity of $100 \%$ in diagnosing of SCSA, as compared to a sensitivity of $93 \%$ for $\mathrm{CT}^{24}$ Both, MRI and CT findings are usually nonspecific, because osteomyelitis, neoplasms, or fluid may show the same signal-decreased T1-weighted images and increased T2-weighted images. ${ }^{4,8}$

Management of SCSA includes antibacterial therapy; however, surgical intervention is often warranted. If the infection is limited on imaging studies, a 4-week antibiotic course is indicated. ${ }^{1}$ If imaging demonstrates mediastinitis or osteomyelitis, antibiotic treatment is recommended for 6 weeks, and surgery is usually indicated as well. Empiric antibiotics should include coverage against $S$. aureus, such as cefazolin or oxacillin. If risk factors for methicillin-resistant Staphylococcus aureus (MRSA) are present (e.g., hemodialysis, intravenous drug use, central line, local prevalence of MRSA), vancomycin, or one of the newer antistaphylococcal antibiotics (linezolid, daptomycin, ceftaroline) should be considered. If the patient is immunocompromised, antibiotics that target gram negative bacteria should be included. Antibiotic therapy may need to be modified based on results of culture and susceptibility testing. ${ }^{20}$

Our case was complicated by the patient having an infection of the pacemaker pocket with the same etiologic agent. When GAS was isolated, the decision was made to leave the pacemaker in place, since the pacemaker pocket was on the contralateral side, and the infection was controlled after initial incision and drainage and antibiotics. We hypothesize that the patient likely became bacteremic early on in his illness and may have developed seeding of the pacemaker wires and/or the pocket. This was not clinically evident at the time of presentation, and only became apparent when antibiotics were discontinued.

\section{Conclusion}

We present, to the best of our knowledge, the first reported case of a group A streptococcal sternoclavicular monoarthritis in an immunocompetent adult. This uncommon location and etiology of infection can delay diagnosis and appropriate treatment, with a possible adverse outcome. A history of antecedent pharyngitis may raise the possibility of GAS infection. Presentation of SCSA is usually subacute, with referred pain to the shoulder, so a high index of suspicion is needed for proper diagnosis. Signs of infection (fever, leukocytosis) may be absent or subtle. Blood cultures, as well as aerobic and anaerobic cultures from the site infection, should be obtained. CT or MRI should be part of a routine evaluation to determine the extent of the disease, and the presence of arthritis requires arthrotomy. Empiric antibiotics should cover $S$. aureus. Caution is needed in a patient with intravascular hardware; if infection involving the hardware is suspected, prolonged antibiotic therapy and removal of the prosthesis should be considered.

\section{Acknowledgements}

The authors thank the Marshfield Clinic Research Foundation's Office of Scientific Writing and Publication for editorial assistance in the preparation of this report.

\section{References}

1. Ross JJ, Shamsuddin H. Sternoclavicular septic arthritis: review of 180 cases. Medicine (Baltimore) 2004;83:139-148.

2. Belzunegui J, Rodríguez-Arrondo F, González C, Queiro R, Martínez de Bujo M, Intxausti JJ, De Dios JR, Figueroa M. Musculoskeletal infections in intravenous drug addicts: report of 34 cases with analysis of microbiological aspects and pathogenic mechanisms. Clin Exp Rheumatol 2000;18:383-386.

3. Guerra C, Spillane LL. Sternoclavicular septic arthritis in a patient with end-stage liver disease. Ann Emerg Med 1996;27:264-266.

4. Gerscovich EO, Greenspan A. Osteomyelitis of the clavicle: clinical, radiologic, and bacterial findings in ten patients. Skeletal Radiol 1994;23:205-210.

5. Blankstein A Nerubay J, Lin E, Keren G, Friedman B, Horoszowski H. Septic arthritis of the sternoclavicular joint. Orthop Rev 1986;15(6):440-44216. Sokoloff L. A prospective necropsy study of arthritis in acquired immunodefficinecy syndrome. Arch Pathol Lab Med 1990; $114: 1035-1037$.

6. Weston VC, Jones AC, Bradbury N, Fawthrop F, Doherty M. Clinical features and outcome of septic arthritis in a single UK health district 1982-1991. Ann Rheumatol Dis 1999;58:214-219.

7. Ely GM: Septic Arthritis of the sternoclavicular joint and osteomyelitis of the proximal clavicle caused by prevotella melaninogenicus: a case with several features delaying diagnosis. J Clin Rheumatol 1999;5:354-359. 
8. Bar-Natan M, Salai M, Sidi Y, Gur H. Sternoclavicular infectious arthritis in previously healthy adults. Semin Arthritis Rheum 2002;32:189-195.

9. Kaandorp CJ, Dinant HJ, van de Laar MA, Moens HJ, Prins AP, Dijkmans BA. Incidence and sources of native and prosthetic joint infection: a community based prospective survey. Ann Rheum Dis 1997;56:470-475.

10. Brancós MA, Peris P, Miró JM, Monegal A, Gatell JM, Mallolas J, Mensa J, García S, Muñoz-Gomez J. Septic arthritis in heroin addicts. Semin Arthritis Rheum 1991:21:81-87.

11. Belzunegui J, Rodríguez-Arrondo F, González C, Queiro R, Martínez de Bujo M, Intxausti JJ, De Dios JR, Figueroa M. Musculoskeletal infections in intravenous drug addicts: report of 34 cases with analysis of microbiological aspects and pathogenic mechanisms. Clin Exp Rheumatol 2000;18:383-386.

12. Crafts RC. A Textbook of Human Anatomy. 3rd ed. New York: John Wiley and Sons; 1985.

13. Jazrawi L, Rokito A, Birdzell M, Zuckerman J. Biomechanics of the Shoulder. 3rd ed. Philadelphia, PA: Lippincott Williams and Wilkins; 2001.

14. Sewell MD, Al-Hadithy N, Le Leu A, Lambert SM. Instability of the sternoclavicular joint: current concepts in classification, treatment and outcomes. Bone Joint $\mathrm{J}$ 2013;95-B:721-731.

15. Silberberg M, Frank EL, Jarrett SR, Silverberg R. Aging and osteoarthritis of the sternoclavicular joint. Am J Pathol 1959; 35:851-865.

16. Nathe T, Tseng S, Yoo B: The anatomy of the supraclavicular nerve during surgical approach to the clavicular shaft. Clin Orthop Relat Res 2011;469:890-894.

17. Hassett G, Barnsley L. Pain referral from the sternoclavicular joint: a study in normal volunteers. Rheumatology (Oxford) 2001;40:859-862.

18. Lewis RP, Sutter VL, Finegold SM. Bone infections involving anaerobic bacteria. Medicine (Baltimore) 1978; 57:279-305.

19. Moyer HR, Ghazi B, Feliciano DV. Sternoclavicular joint infection: a case report. Thorac Cardiovasc Surg 2009;57:500-501.

20. Lowy FD. Staphylococcus aureus infections. N Engl J Med 1998; 339:520-532.

21. Sokoloff L. A prospective necropsy study of arthritis in acquired immunodefficinecy syndrome. Arch Pathol Lab Med 1990;114:1035-1037.

22. Gilad J, Borer A, Riesenberg K, Klein M, Peled N, Schlaeffer F. Polymicrobial polyarticular septic arthritis: a rare clinical entity. Scan J Infect Dis 2001;33:381-383.

23. Chamot AM, Gerster JC. [Sternoclavicular arthritis caused by Streptococcus pyogenes group A complicated by mediastinal abscess in a patient infected with the HIV virus].[Article in French] Rev Rhum Mal Osteoartic 1990;57:663-664.

24. Kendrick AS, Head HD, Rehm J. Management of sternoclavicular joint infections. Am Surg 2007;73:729-732.
Author Affiliations

Radmila M. Savcic-Kos, MD*; Padmavati Mali, MD;

Ajit Abraham, $M D^{\ddagger}$; Meltiady Issa, $M D^{\S}$; Venu Rangu, $M D^{\Uparrow}$; and Rana Nasser, MD\|

*Department of Medicine/Pediatrics Resident, Marshfield, Wisconsin, USA

'Department of Internal Medicine/Hospitalist, Marshfield Clinic, Marshfield, Wisconsin, USA

${ }^{D}$ Department of Internal Medicine/Resident, Marshfield

Clinic, Marshfield, Wisconsin, USA

${ }^{\S}$ Department of Internal Medicine, Mayo Health Systems, Rochester, Minnesota, USA

'Department of Internal Medicine, Mayo Health Systems, Mankato, Minnesota, USA

"Department of Infectious Disease, Marshfield Clinic, Marshfield, Wisconsin, USA 